\title{
Rhodiola crenulata and Its Bioactive Components, Salidroside and Tyrosol, Reverse the Hypoxia-Induced Reduction of Plasma-Membrane-Associated Na,K-ATPase Expression via Inhibition of ROS-AMPK-PKC $\xi$ Pathway
}

\author{
Shih-Yu Lee, ${ }^{1,2}$ Li-Shian Shi, ${ }^{3}$ Hsin Chu, ${ }^{2}$ Min-Hui Li, ${ }^{2}$ Cheng-Wen Ho, ${ }^{2}$ Feng-Yi Lai, \\ Chih-Yang Huang, ${ }^{5,6}$ and Tsu-Chung Chang ${ }^{1,4,5,6}$ \\ ${ }^{1}$ Graduate Institute of Medical Sciences, National Defense Medical Center, Taipei 114, Taiwan \\ ${ }^{2}$ Institute of Aerospace and Undersea Medicine, National Defense Medical Center, Taipei 114, Taiwan \\ ${ }^{3}$ Department of Biotechnology, National Formosa University, Yunlin 632, Taiwan \\ ${ }^{4}$ Department of Biochemistry, National Defense Medical Center, P.O. Box 90048-501, Nei-hu, Taipei 114, Taiwan \\ ${ }^{5}$ Department of Health and Nutrition Biotechnology, Asia University, Taichung 413, Taiwan \\ ${ }^{6}$ Graduate Institute of Basic Medical Science, China Medical University, Taichung 400, Taiwan \\ Correspondence should be addressed to Tsu-Chung Chang; tcchang@ndmctsgh.edu.tw
}

Received 9 March 2013; Revised 5 May 2013; Accepted 20 May 2013

Academic Editor: Evan Paul Cherniack

Copyright (C) 2013 Shih-Yu Lee et al. This is an open access article distributed under the Creative Commons Attribution License, which permits unrestricted use, distribution, and reproduction in any medium, provided the original work is properly cited.

\begin{abstract}
Exposure to hypoxia leads to impaired pulmonary sodium transport, which is associated with Na,K-ATPase dysfunction in the alveolar epithelium. The present study is designed to examine the effect and mechanism of Rhodiola crenulata extract (RCE) and its bioactive components on hypoxia-mediated $\mathrm{Na}, \mathrm{K}$-ATPase endocytosis. A549 cells were exposed to hypoxia in the presence or absence of RCE, salidroside, or tyrosol. The generation of intracellular ROS was measured by using the fluorescent probe DCFH$\mathrm{DA}$, and the endocytosis was determined by measuring the expression level of Na,K-ATPase in the PM fraction. Rats exposed to a hypobaric hypoxia chamber were used to investigate the efficacy and underlying mechanism of RCE in vivo. Our results showed that RCE and its bioactive compounds significantly prevented the hypoxia-mediated endocytosis of Na,K-ATPase via the inhibition of the ROS-AMPK-PKC $\zeta$ pathway in A549 cells. Furthermore, RCE also showed a comparable preventive effect on the reduction of $\mathrm{Na}, \mathrm{K}-\mathrm{ATPase}$ endocytosis and inhibition of AMPK-PKC $\xi$ pathway in the rodent model. Our study is the first to offer substantial evidence to support the efficacy of Rhodiola products against hypoxia-associated Na,K-ATPase endocytosis and clarify the ethnopharmacological relevance of Rhodiola crenulata as a popular folk medicine for high-altitude illness.
\end{abstract}

\section{Introduction}

In normal physiology, there is a homeostasis between alveolar fluid secretion and clearance. Alveolar fluid clearance (AFC) mainly depends on sodium transport in the alveolar epithelium. The apical surface is cleared primarily via the epithelial sodium channel $(\mathrm{ENaC})$, a predominant transepithelial sodium channel in organs including the lungs, kidneys, and colon, which is responsible for $40-60 \%$ of reabsorption. On the other hand, sodium is actively pumped out by $\mathrm{Na}, \mathrm{K}$-ATPase which is located in the basolateral plasma membrane to the pulmonary interstitium. This, in turn, leads to an osmotic gradient in the transepithelial membrane that water follows $[1,2]$. These transporters are essential for the maintenance of transcellular fluid transport. The malfunction of these transporters has been associated with dysfunctions in AFC, alveolar flooding, and edema. For example, alveolar hypoxia, a common phenomenon when abruptly relocating nonacclimatized individuals to a high altitude, is thought to be involved in impaired transalveolar fluid transport. The excessive fluid that subsequently accumulates in alveoli exaggerates alveolar hypoxia and impairs gas exchange, and these 




(a)

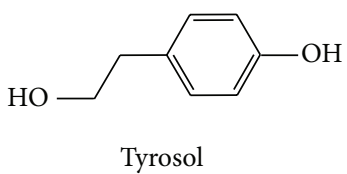

(b)

FIgURE 1: Structures of salidroside (a) and tyrosol (b).

processes are associated with the pathological progression of high altitude pulmonary edema (HAPE), the most lethal form of high-altitude illnesses [3,4].

Recent reports have documented that $\mathrm{Na}, \mathrm{K}$-ATPase plays a key role in the maintenance of AFC. It was shown that both the inhibition and knockdown of Na,K-ATPase expression significantly diminished AFC in rodent models $[5,6]$. In contrast, adenovirus-mediated overexpression of $\mathrm{Na}, \mathrm{K}$ ATPase in alveoli obviously improves AFC in rats [7]. It should be noted that AFC was shown to be associated only with the protein abundance of $\mathrm{Na}, \mathrm{K}$-ATPase in the plasma membrane (PM) rather than the total amount in both cell and rodent models [6-8]. Furthermore, a large body of evidence also indicated that hypoxia is associated with impaired AFC, including mortality in studies using both in vivo and in vitro models as well as clinical studies. The phenomena that manifest during hypoxia are due in part to the hypoxiaassociated malfunction of active sodium transport that results from the declined activity and expression of Na,K-ATPase [9-12].

Exposure to hypoxia leads to mitochondrial complex III dysfunction that results in the intensive generation of intracellular ROS, which in turn activates the AMP-activated protein kinase (AMPK). Through the direct activation of the protein kinase $\mathrm{C} \zeta(\mathrm{PKC} \zeta)$ pathway, the activated AMPK leads to the phosphorylation of $\mathrm{Na}, \mathrm{K}$-ATPase, which serves as the triggering signal for its endocytosis from the basolateral membrane, as indicated in both A549 cells and the alveolar epithelial type II cells of rats $[13,14]$.

Rhodiola species, a popular folk medicine in Asian and eastern European countries, have been used for the prevention of high-altitude illness and are regarded as traditional phytoadaptogens against environmental challenges in Tibet. These species show beneficial properties, including antidepression, neuroprotective, cardioprotective, antifatigue, antihypoxia, and hepatoprotective activities. In addition, Rhodiola species have also been reported to promote longevity and work productivity [15]. Salidroside and tyrosol (Figure 1) were shown to contribute to Rhodiola crenulata's benefits, which include antioxidant, antidepression, antifatigue, and anti-inflammatory activities [16, 17]. In our previous study [18], we demonstrated that Rhodiola crenulata extract (RCE) displayed excellent antioxidant activity and attenuated several indicators of pulmonary edema induced by hypobaric hypoxia in a rodent model. However, the effect and mechanism of Rhodiola crenulata on sodium transport in alveolar cells still needs to be elucidated. The present study attempted to examine whether RCE, salidroside, and tyrosol are efficacious in preventing the hypoxia-mediated Na,K-ATPase endocytosis and to clarify the underlying mechanisms using both A549 cells and rodent models.

\section{Materials and Methods}

2.1. Materials. Rhodiola crenulata were purchased from Chuang Song Zong Pharmaceutical Co., Ltd (Taichung, Taiwan). The plant was authenticated by Dr. H. C. Lin in the National Defense Medical Center, where a voucher specimen (NDMCP no. 1000901) has been deposited. Compound C and GF10923X from TOCRIS (Bristol, UK), N-acetyl-L-cysteine (NAC) from Sigma (St. Louis, MO, USA), and tyrosol, which was a kind gift from Dr. Li-Shian Shi (National Formosa University, Yunlin, Taiwan), were also used in the study.

2.2. Preparation of Salidroside and Rhodiola crenulata Extract. The dried roots of Rhodiola crenulata $(2.0 \mathrm{~kg})$ were ground into a fine powder and soaked $(24 \mathrm{~h})$ with $95 \%$ ethanol $(10 \mathrm{~L} \times 2)$, then refluxed with $95 \%$ ethanol $(10 \mathrm{~L} \times 1)$. The evaporation of the solvent under reduced pressure provided $320.24 \mathrm{~g}$ of RCE, which was successively partitioned between $n$-hexane- $90 \% \mathrm{MeOH}, \mathrm{CHCl}_{3}-\mathrm{H}_{2} \mathrm{O}$, EtOAc- $\mathrm{H}_{2} \mathrm{O}$, and $n$ $\mathrm{BuOH}-\mathrm{H}_{2} \mathrm{O}$ to yield $n$-hexane $(32.89 \mathrm{~g}), \mathrm{CHCl}_{3}(10.08 \mathrm{~g})$, EtOAc (76.15g), $n-\mathrm{BuOH}(101.26 \mathrm{~g})$, and $\mathrm{H}_{2} \mathrm{O}$ (99.86g) fractions. The $n-\mathrm{BuOH}$ fraction $(101.30 \mathrm{~g})$ was applied to Diaion HP-20 column chromatography and eluted with an $\mathrm{H}_{2} \mathrm{O}-\mathrm{MeOH}$ gradient system to give 11 fractions. Fraction 5 (12.34 g) was chromatographed on silica gel and eluted with $\mathrm{CHCl}_{3}-\mathrm{MeOH}(12: 1)$ to give compound $\mathbf{1}(1.24 \mathrm{~g})$. The structure of compound $\mathbf{1}$ was identified as salidroside by comparing it to the spectral data in the literature [19].

2.3. Cell Culture. A549 cells (the human lung adenocarcinoma epithelial cell line), a widely used cell model for the investigation of pulmonary transepithelial sodium transport [14], were purchased from American Type Culture Collection (ATCC, Manassas, VA, USA) and maintained in Dulbecco's modified Eagle medium (DMEM) containing $100 \mathrm{IU} / \mathrm{mL}$ penicillin, $100 \mu \mathrm{g} / \mathrm{mL}$ streptomycin, and $7 \%$ fetal calf serum (Gibco, Gaithersburg, MD, USA) in $5 \% \mathrm{CO}_{2}$ at $37^{\circ} \mathrm{C}$. The medium was changed every two to three days, and the cells were trypsinized and subcultured when the cells were grown 
to approximately $80 \%$ confluence. All of the experiments were carried out using cells with $3-8$ passages. To allow cell differentiation for the experiments, the A549 cells were cultured for another 7 days after reaching confluence.

2.4. Cell Viability Assay. A cell viability assay was performed using a cell counting kit-8 (CCK-8, Dojindo, Japan), as described previously [20]. Briefly, $5 \times 10^{3}$ cells were seeded in each well of the 96-well plates and allowed to attach overnight. Subsequently, the cells were pretreated with different concentrations of RCE, salidroside, or tyrosol for $30 \mathrm{~min}$ before exposure to a hypoxia chamber with a $1 \% \mathrm{O}_{2}, 5 \%$ $\mathrm{CO}_{2}$, and $94 \% \mathrm{~N}_{2}$ hypoxic environment. After $24 \mathrm{~h}$ of hypoxic exposure, the original medium was removed and $10 \mu \mathrm{L}$ of CCK-8 in $90 \mu \mathrm{L}$ of PBS was added to react with cells for $2 \mathrm{~h}$. The absorbance was examined at $450 \mathrm{~nm}$ by using a spectrophotometer.

2.5. Measurement of Intracellular ROS. The quantitation of intracellular ROS was measured using the fluorescent probe 2,7-dichlorofluorescein diacetate (DCFH-DA), as previously described [21]. Briefly, the cells were pretreated with different concentrations of RCE and its bioactive components in a 96well microplate for $30 \mathrm{~min}$ followed by exposure to hypoxia $\left(1 \% \mathrm{O}_{2}\right)$ for $24 \mathrm{~h}$. The original medium was removed, and DCFH-DA in PBS (10 mM) was added to each well at $37^{\circ} \mathrm{C}$ for $15 \mathrm{~min}$ in the incubator. Subsequently, the cells were lysed with lysis buffer and centrifuged at 14,000 rpm for $1 \mathrm{~min}$. The supernatant was collected and measured at an excitation and emission wavelength of 485 and $538 \mathrm{~nm}$, respectively, using a fluorescence microtiter plate reader (POLARstar Galaxy; BMG Labtechnologies, Offenburg, Germany). The results were normalized by the protein concentration of each sample, which was determined by using a BCA (bicinchoninic acid) protein assay kit (Pierce, Rockford, IL, USA).

2.6. PM and Post-Plasma Membrane (PPM) Isolation. The PM fraction of A549 cells and rat lung tissues was isolated as previously described [22]. Briefly, the cells and tissues were lysed in buffer A (50 mM Tris-Cl and $0.5 \mathrm{mM} \mathrm{DTT,} \mathrm{pH} 8.0$ ) supplemented with $0.1 \%(\mathrm{v} / \mathrm{v})$ Nonidet P-40 (NP-40), protease inhibitors, and phosphatase inhibitors. Subsequently, the cell lysate was passed through a $1 \mathrm{~mL}$ syringe fitted with a 25-gauge needle 10 times and centrifuged at $1000 \times \mathrm{g}$ for $10 \mathrm{~min}$. The precipitate was suspended in buffer A without $\mathrm{NP}-40$ and recentrifuged at $1000 \times \mathrm{g}$ for $10 \mathrm{~min}$. Thereafter, the precipitate was resuspended again in buffer A with 1\% NP-40 on ice for $1 \mathrm{~h}$ and then centrifuged at $16000 \times \mathrm{g}$ for $20 \mathrm{~min}$. The supernatant was collected as a PM fraction. In the meantime, the supernatants collected at the first and second steps were pooled and centrifuged at $16000 \times \mathrm{g}$ for $20 \mathrm{~min}$. Thereafter the supernatant was collected as the PPM fraction.

2.7. Hypobaric Hypoxia Exposure in Rats. The method used was described previously [23] and was approved by the Institutional Animal Care and Use Committee of the National Defense Medical Center (IACUC-11-055). The rats were exposed to a simulated altitude of $8000 \mathrm{~m}$ in a hypobaric hypoxia chamber at room temperature for $9 \mathrm{~h}$. The rats were gavaged with RCE suspended in $1 \mathrm{~mL}$ saline via a gastric cannula once daily for 3 days before exposure to hypoxia. The same steps were followed for the normoxic control group (saline). After hypoxic exposure, the rats were anesthetized with an intraperitoneal injection of sodium pentobarbital $(10 \mathrm{mg} / \mathrm{kg})$, the abdomen was opened, and the lung was dissected. The samples were stored at $-80^{\circ} \mathrm{C}$ until use.

2.8. Western Blot Analysis. The expression of specific proteins in A549 cells and rat lung tissues was quantified as described previously [18]. The A549 cells and lung tissue were homogenized in RIPA buffer or buffer A with protease and phosphatase inhibitors. Equal amounts of protein from each sample were separated using sodium dodecyl sulfate (SDS)-10\% polyacrylamide gel electrophoresis (PAGE) and transblotted onto polyvinylidene difluoride (PVDF) membranes (Millipore, Bedford, MA, USA). Immunoblotting was performed with antibodies for $\alpha 1-\mathrm{Na}, \mathrm{K}-\mathrm{ATP}$ ase (Novus Biologicals, Littleton, CO, USA), p-AMPK (T172), AMPK (Cell Signaling Tech, Danvers, MA, USA), PKC $\zeta$ (GeneTex, San Antonio, TX, USA), superoxide dismutase 2 (SOD2), clathrin, glutathione peroxidase 2 (GPx2) (Santa Cruz, CA, USA), and $\beta$-actin (Chemicon, Temecula, CA, USA). The signals were visualized with an enhanced chemiluminescence kit (ECL, Amersham, UK) followed by exposure to X-ray films.

2.9. Statistical Analysis. All of the data represent the mean \pm S.E.M. Significant differences among group means were determined with a one-way ANOVA with repeated measures followed by Newman-Keuls test using SPSS 14.0 for Windows software. $P<0.05$ was considered statistically significant.

\section{Results}

3.1. RCE, Salidroside, and Tyrosol All Suppress the HypoxiaInduced Intracellular ROS Burden with No Significant Cytotoxicity in A549 Cells. After exposure to hypoxia for $24 \mathrm{~h}$, the intracellular ROS level in A549 cells was significantly elevated compared with the control $(1.65 \pm 0.17$-fold, $P<0.001)$ (Figures 2(a), 2(b), and 2(c)). Pretreatment with different concentrations of $\operatorname{RCE}(1.5,3.0,15$, and $30 \mu \mathrm{g} / \mathrm{mL})$ decreased the hypoxia-induced production of intracellular ROS in a dose-dependent manner $(1.27 \pm 0.07,1.20 \pm 0.05,1.11 \pm 0.05$, and 1.09 \pm 0.03 -fold change relative to controls; $P<0.05, P<$ $0.05, P<0.01$, and $P<0.01$, resp.; Figure 2(a)). Consistently, both salidroside and tyrosol $(0.1,1.0,5.0$, and $10 \mu \mathrm{M})$ exerted a similar anti-ROS efficacy in A549 cells under hypoxia in a dose-dependent manner. As shown in Figure 2(b), the results for salidroside are $1.30 \pm 0.08,1.24 \pm 0.06,1.15 \pm 0.04$, and $1.10 \pm 0.03$-fold compared to the control (NS (not significant), $P<0.05, P<0.05$, and $P<0.01$, resp.). Similarly, the results for tyrosol are $1.32 \pm 0.06,1.13 \pm 0.09,1.14 \pm 0.08$, and $1.06 \pm 0.08$-fold compared to the control (NS, $P<$ $0.05, P<0.05$, and $P<0.05$, resp.) (Figure $2(\mathrm{c}))$. As a positive control, NAC (a thiol-reducing agent) also showed 


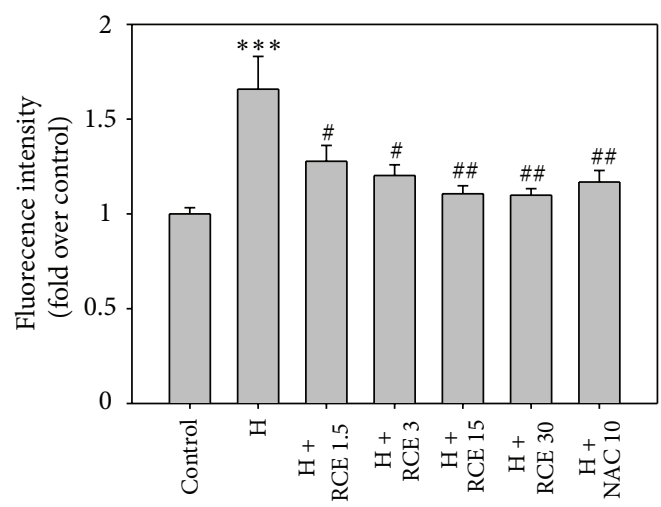

(a)

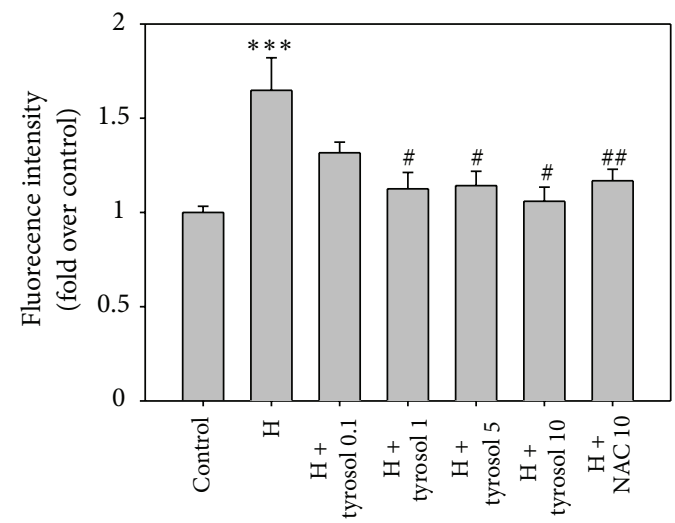

(c)



(e)

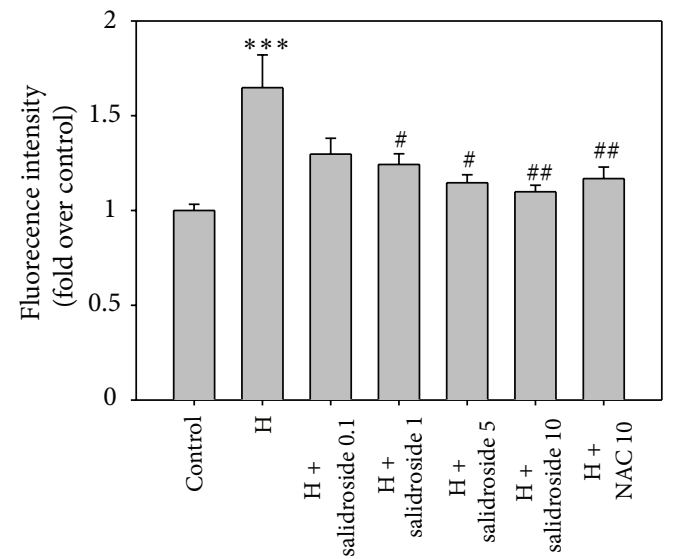

(b)

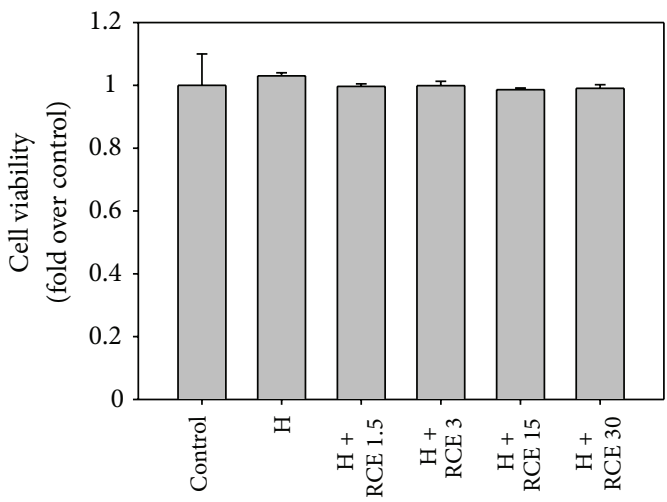

(d)



(f)

FIGURE 2: RCE, salidroside, and tyrosol significantly attenuate the hypoxia-induced generation of intracellular ROS and show no cytotoxicity in A549 cells. The cells were preincubated with the indicated concentrations of RCE, salidroside, and tyrosol for 30 min before hypoxic exposure $\left(1 \% \mathrm{O}_{2}\right)$ for $24 \mathrm{~h}$. The intracellular ROS was measured via the DCFH-DA (10 mM) fluorescence method ((a), (b), and (c)). The cell viability under hypoxia in the presence or absence of Rhodiola products was examined using a CCK-8 reagent kit ((d), (e), and (f)). The concentration units of RCE, salidroside, and tyrosol are $\mu \mathrm{g} / \mathrm{mL}$ and $\mu \mathrm{M}$, respectively. NAC $(10 \mu \mathrm{M})$ served as a positive control. The results represent the mean \pm SEM of six independent experiments. ${ }^{*} P<0.05 ;{ }^{* *} P<0.01 ;{ }^{* * *} P<0.001$ versus control; ${ }^{\#} P<0.05$; ${ }^{\# \#} P<0.01$; ${ }^{\# \# \#} P<0.001$ versus hypoxia $(\mathrm{H})$. 
the ability to alleviate the hypoxia-induced production of intracellular ROS $(1.17 \pm 0.06$-fold compared to the control, $P<0.01)$. Furthermore, RCE, salidroside, and tyrosol showed no significant cytotoxicity in the dose range studied under our experimental conditions (Figures 2(d), 2(e), and 2(f)).

\subsection{RCE, Salidroside, and Tyrosol Prevent Hypoxia-Mediated} Na,K-ATPase Endocytosis in A549 Cells. To identify whether the method we used is applicable to separate the PM and PPM fractions, $\alpha 1-\mathrm{Na}, \mathrm{K}-\mathrm{ATPase}$ and $\beta$-actin were used as the specific protein markers for the PM and PPM fractions, respectively. The results shown in Figure 3(a) indicate that the two fractions were clearly separated, as there is no $\beta$-actin in the PM and the $\alpha 1-\mathrm{Na}, \mathrm{K}-\mathrm{ATP}$ ase is predominantly in the PM fraction. Exposure to hypoxia significantly decreased the abundance of the $\alpha 1-\mathrm{Na}, \mathrm{K}-\mathrm{ATPase}$ in the PM in A549 cells (0.54 \pm 0.04 -fold compared to control; $P<0.01$, in Figures 3(b), 3(c), and 3(d)); pretreatment with RCE at different doses $(15$ and $30 \mu \mathrm{g} / \mathrm{mL}$ ) effectively blunted the $\alpha 1-\mathrm{Na}, \mathrm{K}$-ATPase endocytosis in response to hypoxia $(0.85 \pm 0.09$ and $0.70 \pm$ 0.17-fold compared to the control, in Figure 3(b)) with a significant difference at the dose of $15 \mu \mathrm{g} / \mathrm{mL}(P<0.05)$. Salidroside $(1.0$ and $5.0 \mu \mathrm{M})$ also showed similar efficacy for the reduction of $\alpha 1-\mathrm{Na}, \mathrm{K}-\mathrm{ATPase}$ in the PM under hypoxia $(0.90 \pm 0.16$ and $0.91 \pm 0.10$-fold compared to the control $)$ with optimal efficacy at a dose of $5.0 \mu \mathrm{M}(P<0.05)$. Similarly, tyrosol, which is the aglycone of salidroside, exerted potent protective effects comparable to salidroside $(0.83 \pm 0.02$ and $0.87 \pm 0.03$-fold compared to control), especially at doses of 1.0 and $5.0 \mu \mathrm{M}(P<0.05)$. NAC $(10 \mu \mathrm{M})$ was used as the positive control and displayed a potent inhibitory effect on the hypoxia-induced $\alpha 1-\mathrm{Na}, \mathrm{K}-\mathrm{ATP}$ ase endocytosis as expected $(0.83 \pm 0.10$-fold compared to control, $P<0.05)$. To investigate the underlying mechanisms of the effect of RCE, salidroside, and tyrosol on hypoxia-induced Na,K-ATPase endocytosis in A549 cells, we assessed the expression of PKC $\zeta$ in the PM in response to hypoxia. As shown in Figures 3(b), $3(\mathrm{c})$, and $3(\mathrm{~d})$, the hypoxia-mediated decrease in $\alpha 1-\mathrm{Na}, \mathrm{K}-$ ATPase is accompanied by the increased translocation of PKC $\zeta$ to the PM $(1.37 \pm 0.09$-fold compared to control; $P<$ 0.05). Meanwhile, we also observed that hypoxia significantly increased the phosphorylation of AMPK at T172 in the PPM fraction $(2.29 \pm 0.23$-fold compared to control; $P<0.01)$. Furthermore, the protein abundance of $\alpha 1-\mathrm{Na}, \mathrm{K}$-ATPase was found to be increased in the PPM fractions under hypoxia (2.06 \pm 0.16 -fold compared to control; $P<0.01$, in Figures $3(\mathrm{~b}), 3(\mathrm{c})$, and $3(\mathrm{~d}))$. However, preincubation with RCE (15 and $30 \mu \mathrm{g} / \mathrm{mL}$ ) ameliorated both the $\alpha 1-\mathrm{Na}, \mathrm{K}-\mathrm{ATPase}$ endocytosis and the translocation of PKC $\zeta$ to the PM $(1.09 \pm 0.03$ and $1.05 \pm 0.01$-fold compared to control, resp., $P<0.05)$ in addition to attenuating the increased level of phosphorylated AMPK (p-AMPK) in response to hypoxia (1.29 \pm 0.10 and $1.16 \pm 0.03$-fold compared to control, resp., $P<0.05$ and $P<$ 0.01 ; Figure 3(b)). Moreover, salidroside and tyrosol (1.0 and $5.0 \mu \mathrm{M})$ also showed similar efficacies in alleviating increased p-AMPK $(1.30 \pm 0.05$ and $1.25 \pm 0.04$-fold compared to control, resp., $P<0.05 ; 1.30 \pm 0.10$ and $1.11 \pm 0.07$-fold compared to control, resp., $P<0.05$ and $P<0.01)$ and PKC $\zeta$ translocation $(1.08 \pm 0.03$ and $1.06 \pm 0.02$-fold compared to control, resp., $P<0.05 ; 1.03 \pm 0.04$ and $1.03 \pm 0.01$-fold compared to control, resp., $P<0.05$ ) under hypoxia (Figures 3(b) and 3(c)). Consistently, NAC also showed significant efficacy in attenuating translocated $\mathrm{PKC} \zeta(1.02 \pm 0.03$-fold compared to control; $P<0.05)$ and increased p-AMPK $(1.13 \pm 0.06-$ fold compared to control; $P<0.01$ ) in response to hypoxia. On the other hand, there was no significant inductive effect on major cellular antioxidant enzymes, including SOD2 and GPx2, under hypoxic conditions, despite the fact that these enzymes were reported to provide beneficial effects for $\mathrm{Na}, \mathrm{K}$ ATPase against hypoxic insults [8].

3.3. The Protective Effects of RCE, Salidroside, and Tyrosol Are Associated with Alleviating AMPK-PKCל Pathway under Hypoxia. We observed that hypoxia altered the expression of $\alpha 1-\mathrm{Na}, \mathrm{K}-\mathrm{ATP}$ ase in both PM and PPM fractions (Figures 3(b), 3(c), and 3(d)); however, the total amount of $\alpha 1-\mathrm{Na}, \mathrm{K}$ ATPase in the cell lysate remained unchanged in response to hypoxia (Figures 4(a), 4(b), and 4(c)). The results confirmed that hypoxia indeed triggered $\alpha 1-\mathrm{Na}, \mathrm{K}$-ATPase endocytosis from the PM to intracellular compartments. We also showed that the effects of Rhodiola products are specific for hypoxia-induced Na,K-ATPase endocytosis, because they show no significant changes on $\alpha 1-\mathrm{Na}, \mathrm{K}$-ATPase expression in both PM and the total cell lysate (TCL) under normoxia (Figures 4(d), 4(e), and 4(f)). To further clarify the signal mechanisms of RCE and its bioactive compounds for the preservation of $\alpha 1-\mathrm{Na}, \mathrm{K}-\mathrm{ATPase}$ through the ROS-AMPKPKC $\zeta$ pathway, the effect of hydrogen peroxide $\left(\mathrm{H}_{2} \mathrm{O}_{2}\right)$ was investigated under normoxia. We found that $\mathrm{H}_{2} \mathrm{O}_{2}$ $(100 \mu \mathrm{M})$ significantly decreased the abundance of $\alpha 1-\mathrm{Na}, \mathrm{K}-$ ATPase in PM in normoxia (0.60 \pm 0.02 -fold compared to control; $P<0.01)$. However, RCE, salidroside, and tyrosol effectively prevented the $\mathrm{Na}, \mathrm{K}$-ATPase endocytosis $(0.82 \pm 0.04,0.94 \pm 0.06$, and $0.92 \pm 0.06$-fold compared to control, resp., $P<0.05)$ response to application of $\mathrm{H}_{2} \mathrm{O}_{2}$ in normoxia (Figure $4(\mathrm{~g})$ ). Furthermore, the effect of compound C (an AMPK inhibitor), AICAR (5-amino-1- $\beta$ D-ribofuranosyl-imidazole-4-carboxamide, an AMPK activator), and GF10923X (a PKC inhibitor) were also examined. Pre-incubation with AICAR abolished the protective effect of RCE, salidroside, and tyrosol on the hypoxia-mediated $\alpha 1$ $\mathrm{Na}, \mathrm{K}-\mathrm{ATPase}$ endocytosis $(0.64 \pm 0.02,0.60 \pm 0.02$ and $0.59 \pm$ 0.01 -fold compared to control, resp.; $P<0.001$ ), which was accompanied by increased $\mathrm{p}-\mathrm{AMPK}$ and $\mathrm{PKC} \zeta$ translocation in the PM fraction (Figure 4(i)). In addition, pretreatment of the cells with compound C or GF10923X effectively attenuated the $\alpha 1-\mathrm{Na}, \mathrm{K}-\mathrm{ATP}$ ase endocytosis $(0.76 \pm 0.03$ and $0.72 \pm 0.05$-fold compared to control, resp.; $P<0.05$ and $P=0.11)$ via the suppression of $\mathrm{p}$-AMPK or translocated $\mathrm{PKC} \zeta$, respectively (Figure $4(\mathrm{k})$ ). These results indicate that increased $\mathrm{p}$-AMPK and translocated $\mathrm{PKC} \zeta$ are required for the endocytosis of $\mathrm{Na}, \mathrm{K}$-ATPase under hypoxic conditions in A549 cells.

3.4. RCE Confers a Similar Preventive Effect and Mechanism on Hypoxia-Mediated Reduction of Na,K-ATPase in a Hypobaric Hypoxia Rat Model. To verify the effect of RCE in vivo, a hypobaric hypoxia rat model was employed. The results 




(a)

(b)


Salidroside $(\mu \mathrm{M})$, hypoxia



(c)

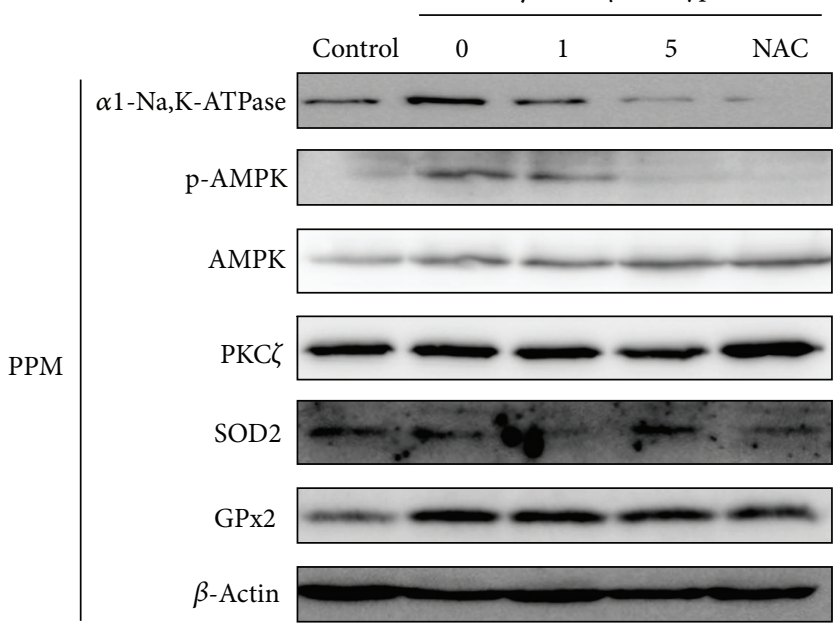

(d)

Figure 3: Continued. 


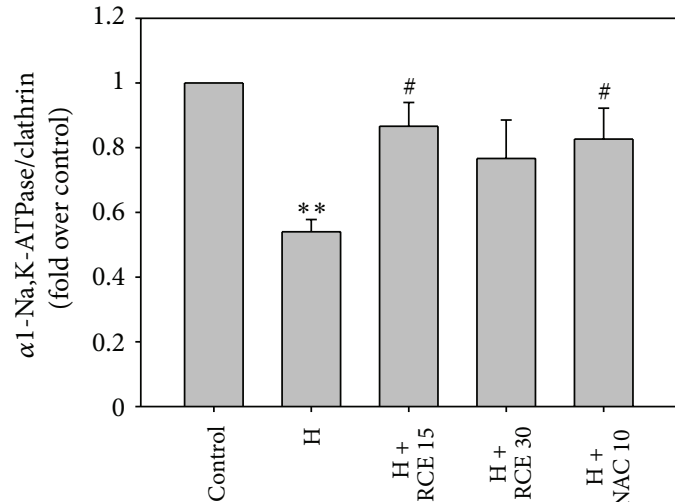

(e)

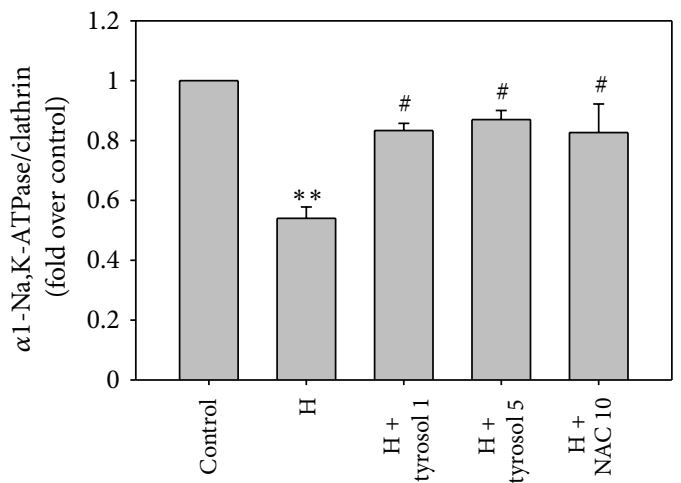

(g)

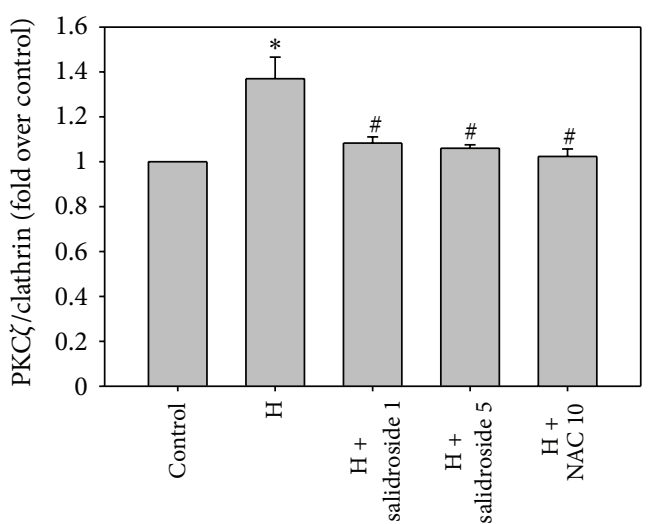

(i)

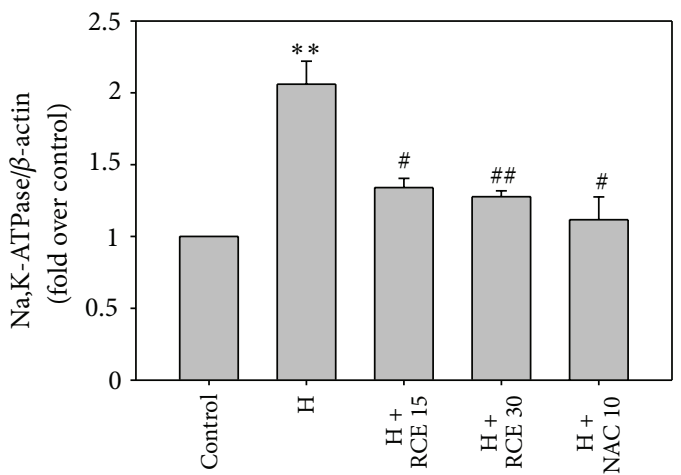

(k)

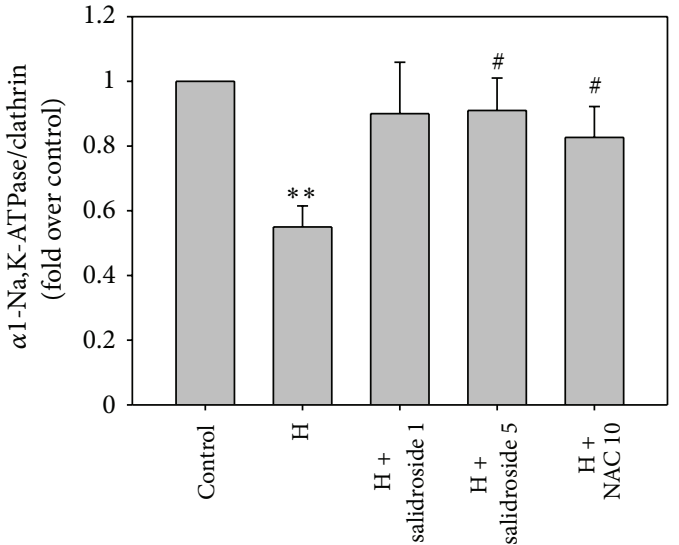

(f)

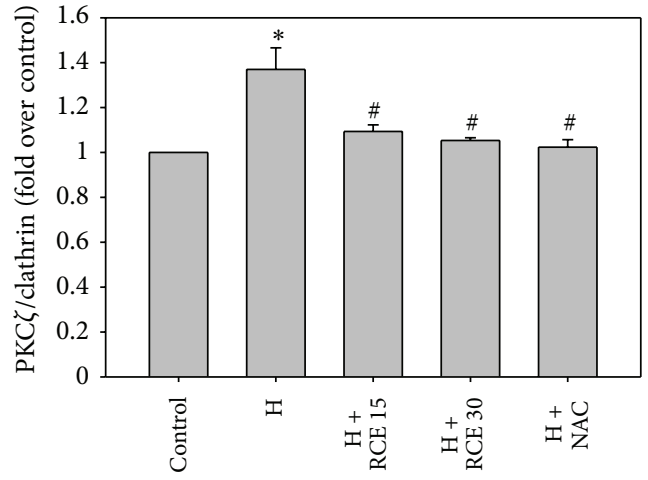

(h)

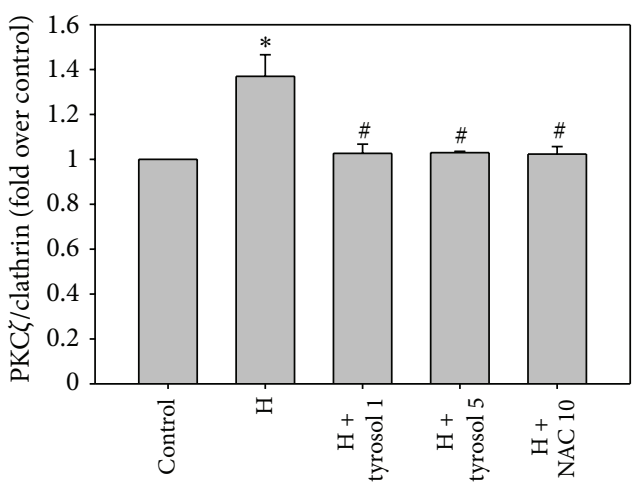

(j)

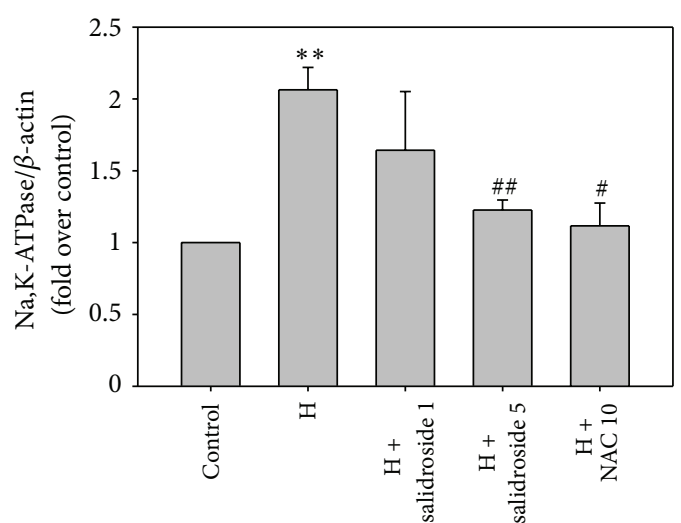

(l)

Figure 3: Continued. 


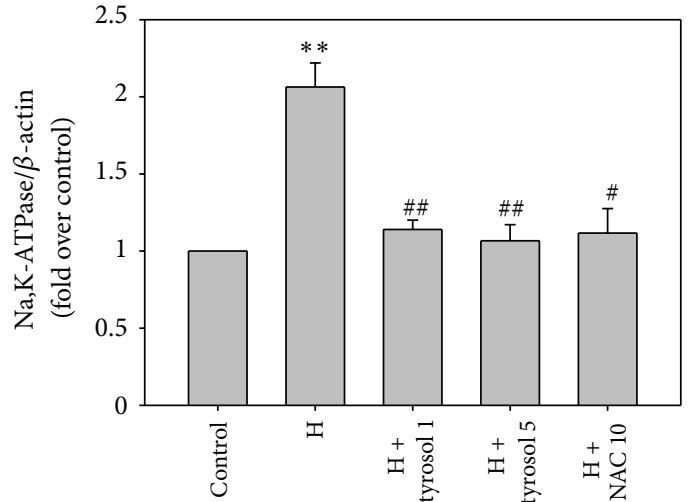

$(\mathrm{m})$



(o)

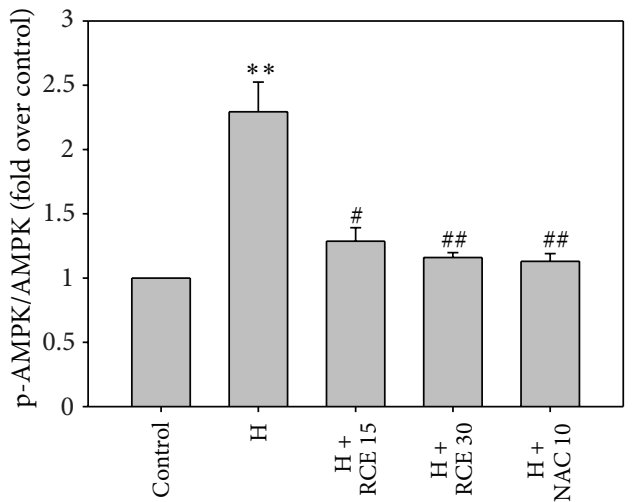

(n)

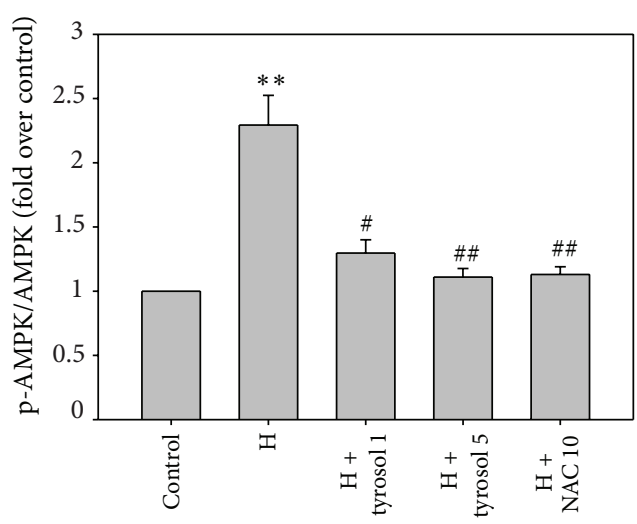

(p)

FIGURE 3: RCE, salidroside, and tyrosol prevent hypoxia-mediated Na,K-ATPase endocytosis in A549 cells. The analysis of the distribution of $\alpha 1-\mathrm{Na}, \mathrm{K}-\mathrm{ATP}$ ase and $\beta$-actin in the plasma membrane (PM) and post-plasma membrane (PPM) fractions by western blotting (a). The A549 cells were exposed to hypoxia $\left(1 \% \mathrm{O}_{2}\right)$ for $24 \mathrm{~h}$, and the expressions of $\alpha 1-\mathrm{Na}, \mathrm{K}-\mathrm{ATP}$ ase, PKC $\zeta$, p-AMPK, SOD2, and GPx2 were analyzed with or without a 30 min pretreatment with RCE (b), salidroside (c), and tyrosol (d) in the PM and PPM. Clathrin served as a loading control. (e)-(g) and (h)-(j) are the quantitative data for the relative levels of $\alpha 1-\mathrm{Na}, \mathrm{K}-\mathrm{ATPase}$ and PKC $\zeta$ in the PM from (b), (c), and (d). (k)-(m) and $(\mathrm{n})-(\mathrm{p})$ are the quantitative data for the relative levels of $\alpha 1-\mathrm{Na}, \mathrm{K}-\mathrm{ATP}$ ase and p-AMPK in the PPM from (b), (c), and (d). The results represent the mean \pm SEM of three independent experiments. ${ }^{*} P<0.05 ;{ }^{* *} P<0.01 ;{ }^{* * *} P<0.001$ versus control; ${ }^{\#} P<0.05$; ${ }^{\# \#} P<0.01$; $\#$ \#\# $P 0.001$ versus hypoxia $(\mathrm{H})$.

indicate a significantly decreased protein abundance of $\alpha 1-$ $\mathrm{Na}, \mathrm{K}$-ATPase in both the PM $(0.53 \pm 0.11$-fold over control with $P<0.01$, Figures 5(a) and 5(b)) and PPM $(0.54 \pm 0.10-$ fold over control with $P<0.01$, Figures 5(d) and 5(e)) fractions of rat lung tissue lysates in response to hypoxia. Hypoxia also significantly increased the level of PKC $\zeta$ in the PM $(1.51 \pm 0.04$-fold compared to control with $P<0.001$, Figures 5(a) and 5(c)), which was accompanied by increased p-AMPK $(2.78 \pm 0.40$-fold compared to control with $P<0.01$, Figures 5(d) and 5(f)) similar to the results observed in A549 cells. Nevertheless, pretreatment with RCE (50 or $100 \mu \mathrm{g} / \mathrm{kg}$ ) effectively alleviated both the PKC $\zeta$ translocation in the PM (1.18 \pm 0.10 and $1.17 \pm 0.07$-fold compared to control, resp., $P<0.05)$ and the increased p-AMPK levels $(1.42 \pm 0.25$ and $1.35 \pm 0.27$-fold over control, resp., $P<0.05)$. RCE also preserved the abundance of $\alpha 1-\mathrm{Na}$,K-ATPase in both the PM $(0.90 \pm 0.07$ and $0.92 \pm 0.09$-fold over control, resp., $P<0.05)$ and PPM $(0.83 \pm 0.04$ and $0.85 \pm 0.05$-fold over control, resp., $P<0.05)$ during hypoxia.

\section{Discussion}

Salidroside and tyrosol are regarded as the major bioactive compounds and are used as the standard markers for the quantitative analysis of the quality of different Rhodiola species or preparations [24]. Until now, most studies have focused on the role of salidroside, rather than tyrosol, in the efficacy of Rhodiola species. Thus, intensive studies about tyrosol are still lacking. In this study, we found that tyrosol, an antioxidant phenolic compound, showed antioxidant properties comparable to those of salidroside in the regulation of Na,K-ATPase via similar protective mechanisms. These results suggest that tyrosol may play a significant role in the protective effects of Rhodiola crenulata against oxidativestress-associated disorders. It should be noted that tyrosol also exists in wine and olive oils, two representative components of the Mediterranean diet, which have been reported to have antioxidant, anticancer, and cardiovascular benefits [25, 26]. Thus, it is interesting to investigate whether salidroside 


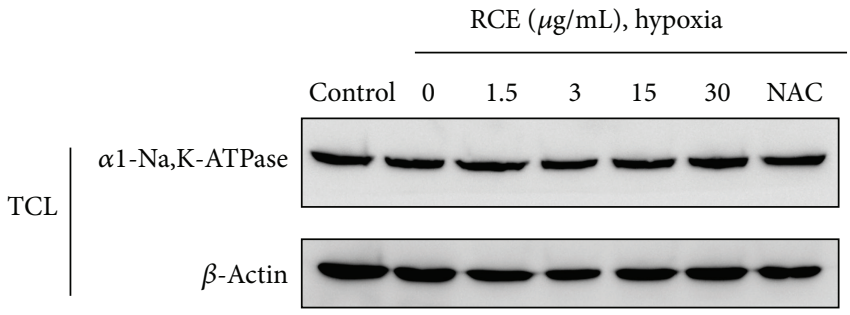

(a)

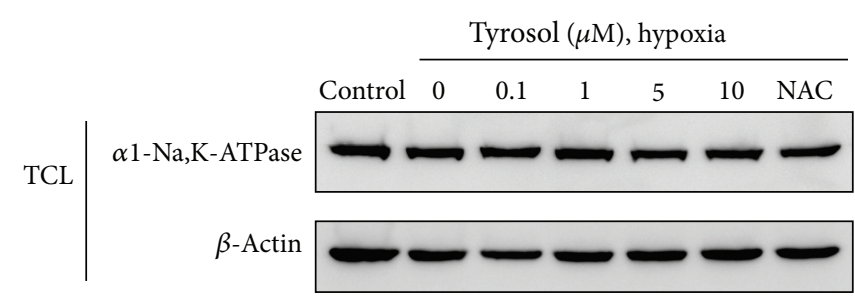

(c)

Salidroside $(\mu \mathrm{M})$, normoxia

$\begin{array}{llllll}\text { Control } & 0.1 & 1 & 5 & 10 & \text { NAC }\end{array}$
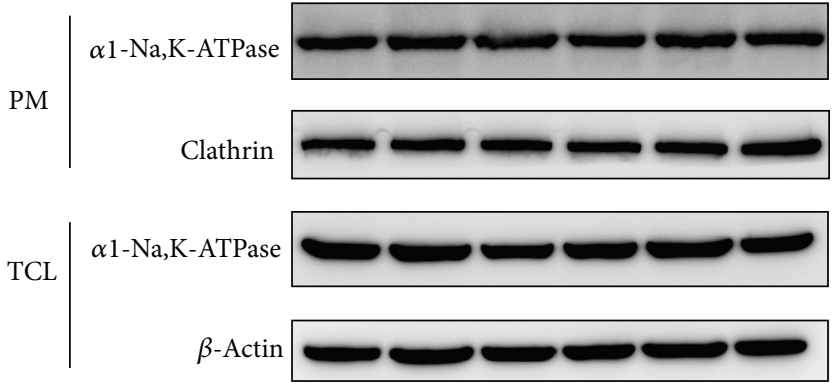

(e)

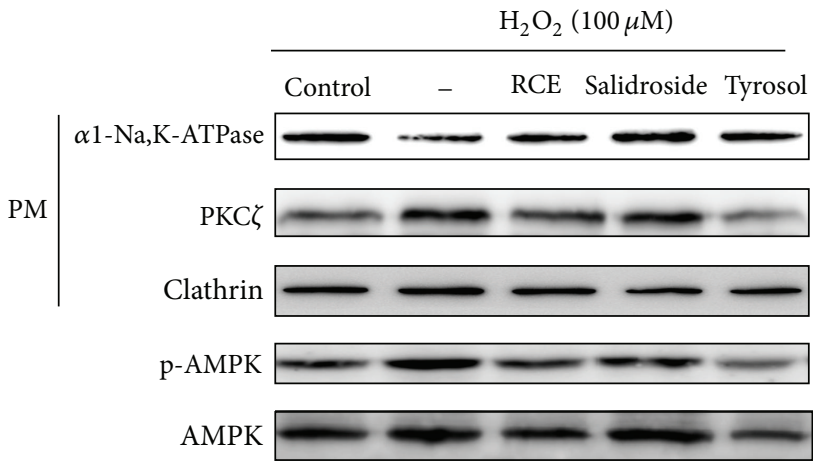

(g)

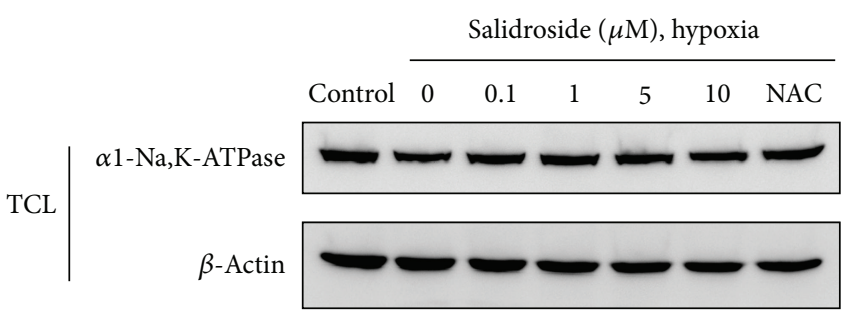

(b)

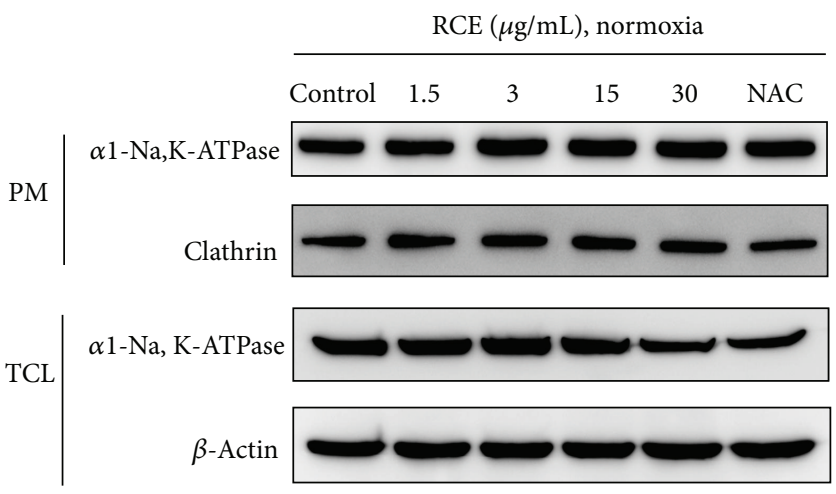

(d)

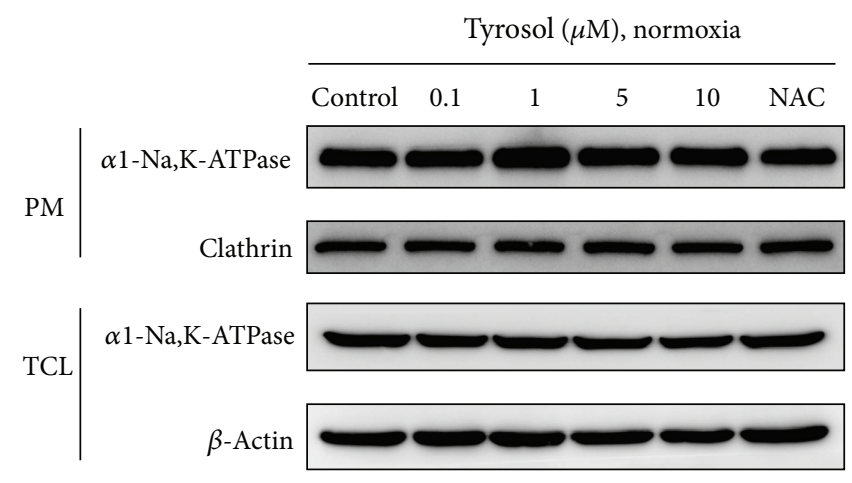

(f)



(h)

FIgURE 4: Continued. 


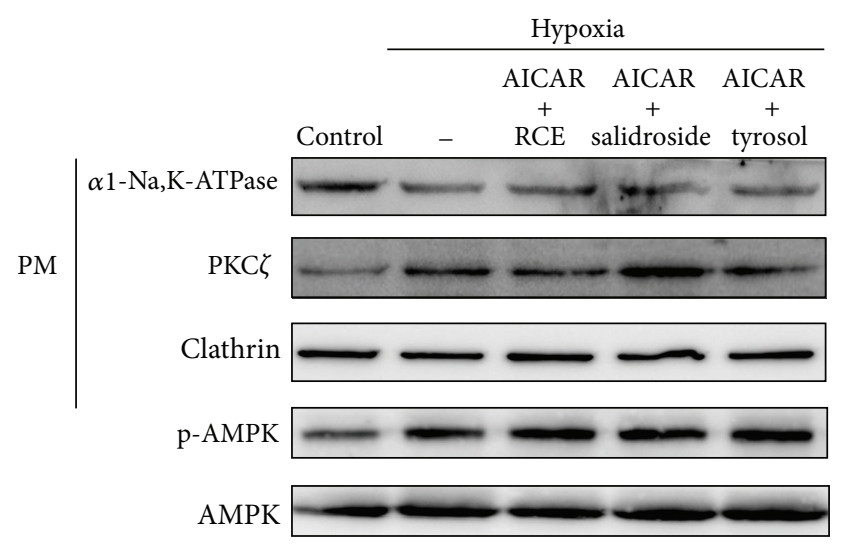

(i)



$(\mathrm{k})$

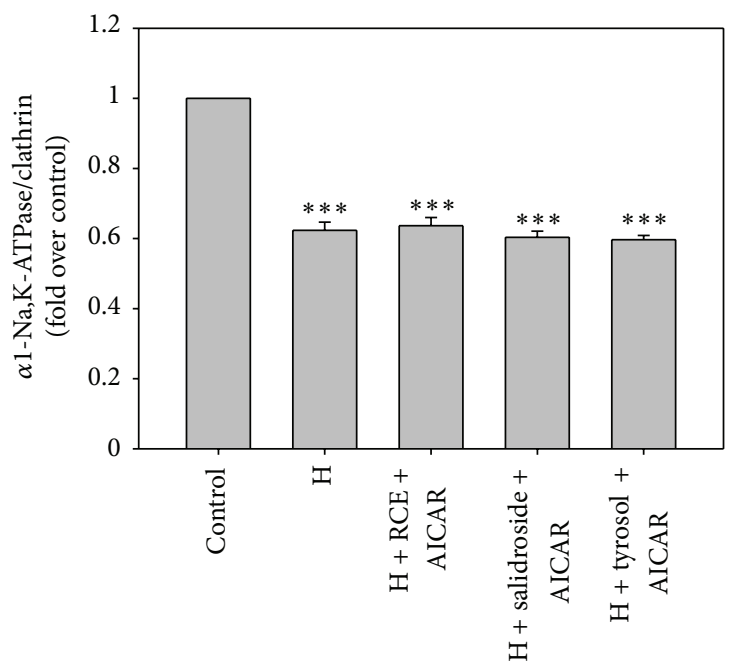

(j)

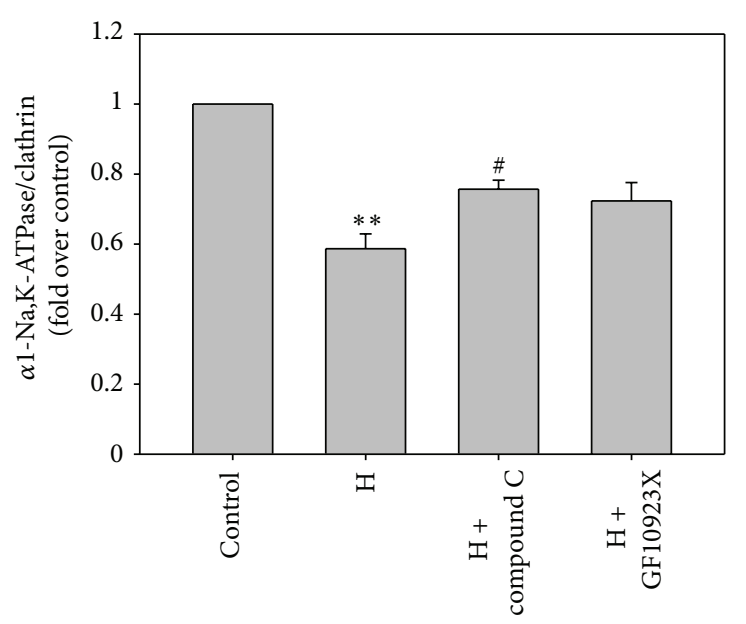

(1)

FIGURE 4: The protective effects of RCE, salidroside, and tyrosol are associated with alleviating the AMPK-PKC $\zeta$ pathway under hypoxia. The cells were pretreated for $30 \mathrm{~min}$ with the indicated concentrations of Rhodiola products or chemicals under hypoxia $\left(1 \% \mathrm{O}_{2}\right)$ or normoxia for $24 \mathrm{~h}$. The expressions of $\alpha 1-\mathrm{Na}, \mathrm{K}-\mathrm{ATP}$ ase were analyzed in the presence or absence of RCE (a), salidroside (b), and tyrosol (c) in total cell lysate (TCL) under hypoxia or RCE (d), salidroside (e), and tyrosol (f) in both PM and TCL under normoxia. Cells were pretreated with RCE $(15 \mu \mathrm{g} / \mathrm{mL})$, salidroside $(5.0 \mu \mathrm{M})$, or tyrosol $(5.0 \mu \mathrm{M})$ for $30 \mathrm{~min}$ and then exposed to $\mathrm{H}_{2} \mathrm{O}_{2}(100 \mu \mathrm{M})$ for another 30 min under normoxia (g). AICAR $(2 \mathrm{mM})$ was added in combination with RCE $(15 \mu \mathrm{g} / \mathrm{mL})$, salidroside $(5.0 \mu \mathrm{M})$, or tyrosol (5.0 $\mu \mathrm{M})$ (i). Compound C $(2 \mu \mathrm{M})$ or GF10923X $(1 \mu \mathrm{M})$ was preincubated alone prior to hypoxic exposure $(\mathrm{k})$. (h), (j), and (l) are the quantitative data for the relative levels of $\alpha 1-\mathrm{Na}, \mathrm{K}-\mathrm{ATP}$ ase in the PM from (g), (i), and (k). The results represent the mean \pm SEM of three independent experiments. ${ }^{*} P<0.05$; ${ }^{* *} P<0.01 ;{ }^{* * *} P<0.001$ versus control; ${ }^{\#} P<0.05 ;{ }^{\# \#} P<0.01 ;{ }^{\# \#} P<0.001$ versus hypoxia $(\mathrm{H})$ or $\mathrm{H}_{2} \mathrm{O}_{2}$.

and tyrosol have any beneficial effect for the prevention of cancer or cardiovascular disease.

The present study shows for the first time that salidroside and tyrosol exert preventive effects on $\alpha 1-\mathrm{Na}, \mathrm{K}$-ATPase endocytosis in A549 cells during hypoxia with efficacies comparable to RCE. We also clarified the underlying mechanisms of RCE and its bioactive components by showing that they are associated with counteracting the ROS-AMPK$\mathrm{PKC \zeta}$ pathway. Using a hypobaric hypoxia rodent model, we further found that hypoxia-induced molecular responses similar to those shown in A549 cells and RCE exerted similar protective effects via similar mechanisms. A549, which displays many characteristics of alveolar epithelial cells, is a generally accepted model of ion transport which displays great similarity to the primary rat alveolar type II (ATII) cells in response to hypoxia $[27,28]$. The trafficking of $\mathrm{Na}, \mathrm{K}$-ATPase under hypoxia is endocytosis from the plasma membrane to intracellular compartments first and 


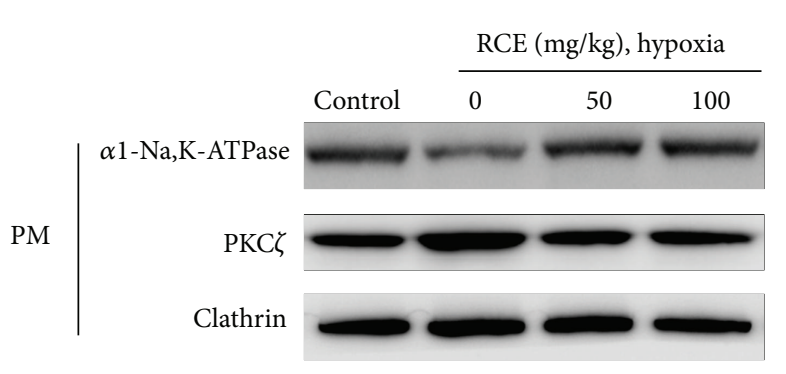

(a)

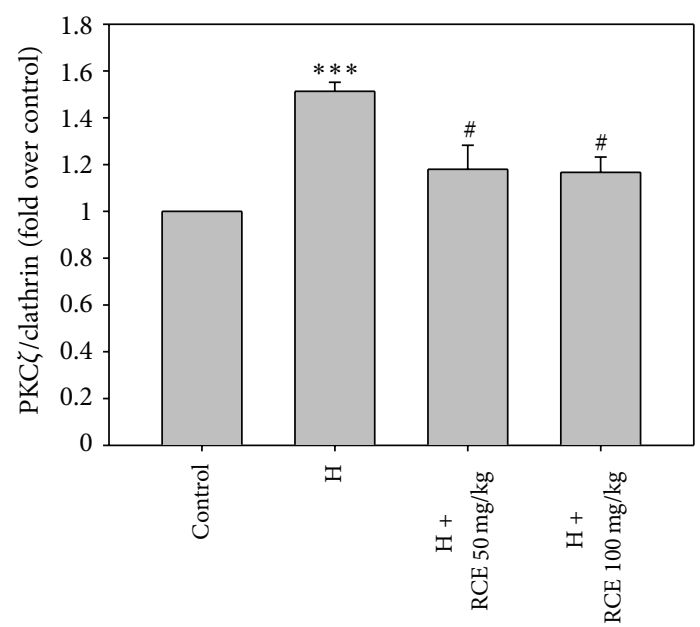

(c)

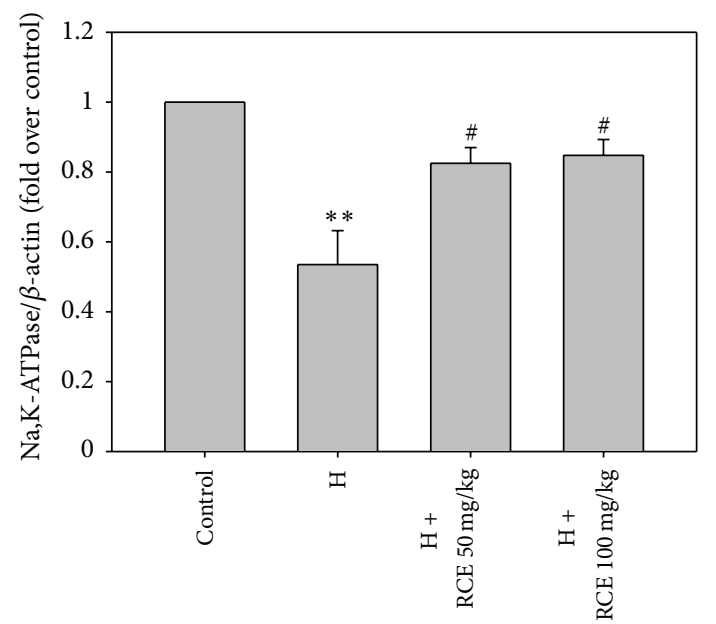

(e)



(b)



(d)

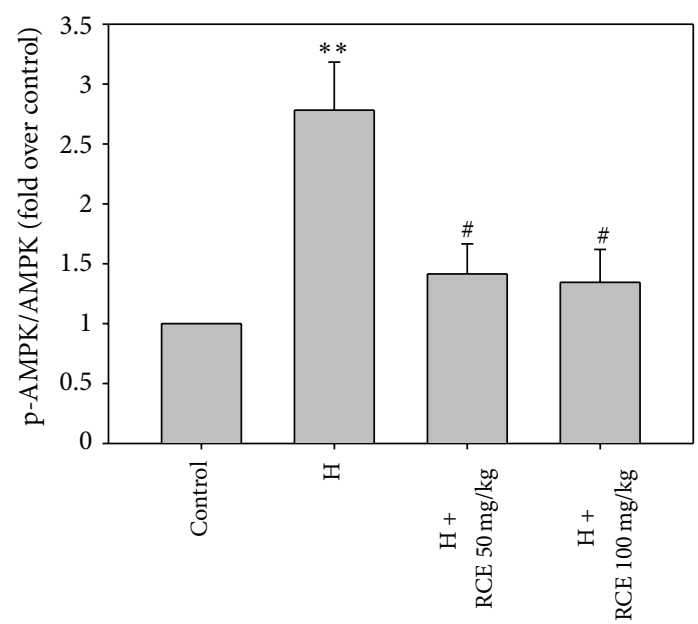

(f)

FIGURE 5: RCE confers a similar preventive effect on the hypoxia-mediated reduction of Na,K-ATPase via a similar mechanism in a hypobaric rodent model. The rats were exposed to simulated hypobaric hypoxia $(8000 \mathrm{~m})$ for $9 \mathrm{~h}$ in the presence or absence of RCE. The expression of $\alpha 1-\mathrm{Na}, \mathrm{K}-\mathrm{ATP}$ ase and PKC $\zeta$ in the PM isolated from the right upper lobe of the lung (a) and the relative levels of $\alpha 1-\mathrm{Na}, \mathrm{K}-\mathrm{ATPase}(\mathrm{b})$ and PKC $\zeta$ (c) were quantified. The expression of $\alpha 1-\mathrm{Na}, \mathrm{K}-\mathrm{ATPase}$ and $\mathrm{p}$-AMPK in the PPM (d) and the relative levels of $\alpha 1-\mathrm{Na}, \mathrm{K}-\mathrm{ATPase}$ (e) and p-AMPK (f) were quantified. The results represent the mean \pm SEM of four independent experiments. ${ }^{*} P<0.05$; ${ }^{* *} P<0.01$; ${ }^{* * *} P<0.001$ versus control; ${ }^{\#} P<0.05 ;{ }^{\#} P<0.01 ;{ }^{\# \# \#} P<0.001$ versus hypoxia $(\mathrm{H})$. 
then degradation [1]. Our results showed more rapid $\mathrm{Na}, \mathrm{K}$ ATPase degradation under hypoxia in rat lungs than in A549 cells (Figures 5(a) and 5(d)), which is consistent with previous studies $[8,29]$. The results suggest that different rates of Na-K-ATPase degradation in response to hypoxia may exist between A549 cells and rat ATII cells. Even so, $\mathrm{Na}, \mathrm{K}-\mathrm{ATPase}$ endocytosis in both A549 and rat ATII cells is primarily through ROS-AMPK-PKC $\zeta$ pathways [14, 30]. Thus, the results of A549 cells should be generally transferable to the in vivo situation.

Previous evidence indicated that diminishing mitochondrial ROS via antioxidants or mitochondrial inhibitors prevented hypoxia-induced decreases in both the expression and activity of $\mathrm{Na}, \mathrm{K}$-ATPase [13]. In the present study, we show that RCE and its bioactive components significantly alleviated both the production of intracellular ROS and Na,K-ATPase endocytosis in response to hypoxia. Furthermore, we also ruled out the possibility that the reduction of intracellular ROS burden was due to cytotoxicity imparted by RCE, salidroside, and tyrosol. In addition, we also showed that Rhodiola products all significantly prevented $\mathrm{H}_{2} \mathrm{O}_{2}$-induced $\alpha 1$ $\mathrm{Na}, \mathrm{K}$-ATPase endocytosis (Figure $4(\mathrm{~g})$ ). The anti-ROS effect of these Rhodiola products is consistent with previous studies that found that salidroside is able to decrease the oxidative stress induced by $\mathrm{CoCl}_{2}$, which is a hypoxia-mimicking agent, and showed protective effects against $\mathrm{H}_{2} \mathrm{O}_{2}$ insults in different cell lines $[31,32]$. Combined with the previous findings, our results demonstrate that Rhodiola products exhibit potency in alleviating oxidative stress in different hypoxic models. The antioxidant NAC, which is used as a positive control in this study, showed antioxidant activity to alleviate the activation of the AMPK-PKC $\zeta$ pathway and $\alpha 1-\mathrm{Na}, \mathrm{K}-\mathrm{ATPase}$ endocytosis under hypoxic conditions. Furthermore, NAC was also reported to attenuate oxidative stress and preserved $\mathrm{Na}, \mathrm{K}$-ATPase activity in an oleic-acid-induced lung injury rodent model due to its antioxidant properties [33]. Thus, our findings suggest that the protective effect of these Rhodiola products is associated with their antioxidant activity. These results also imply that supplementation with antioxidants might help people working at high altitudes maintain AFC.

AMPK is a critical metabolic sensor for energy homeostasis that regulates a large number of downstream targets. AMPK has also been shown to play a key role in the regulation of $\mathrm{Na}, \mathrm{K}$-ATPase endocytosis via the phosphorylation and recruitment of PKC $\zeta$ during hypoxia [14]. To identify the role of AMPK in the protective effects of Rhodiola products, the effects of AICAR and compound C were examined in the present study. Our results showed that compound C completely inhibited p-AMPK and alleviated the $\alpha 1-\mathrm{Na}, \mathrm{K}-\mathrm{ATP}$ ase endocytosis in response to hypoxia (Figure $4(\mathrm{k})$ ). In contrast, AICAR abolished the protective effect of RCE, salidroside, and tyrosol, which was accompanied by increased p-AMPK levels and PKC $\zeta$ translocation. In addition to the AMPK signal modulators, pretreatment with GF10923X also inhibited the translocation of PKC $\zeta$ to PM and ameliorated hypoxia-mediated $\alpha 1-\mathrm{Na}, \mathrm{K}$-ATPase endocytosis (Figure 4(i)). It should be noted that GF10923X did not inhibit the level of $\mathrm{p}$-AMPK, confirming that the translocation of $\mathrm{PKC} \zeta$ is a downstream consequence of AMPK phosphorylation under hypoxia. The findings suggest that Rhodiola products prevented the hypoxia-induced $\mathrm{Na}, \mathrm{K}$ ATPase endocytosis mediated by the AMPK-PKC $\zeta$ pathway. However, the effects of Rhodiola products for AMPK are controversial. Rhodiola rosea and salidroside were shown to activate the AMPK pathway to regulate the cellular energy status in different cell lines $[34,35]$. This implied that Rhodiola products may trigger energy production by activating AMPK when organisms are in low-energy stress. In contrast, the present study shows that RCE and its bioactive components all attenuated hypoxic stress and intracellular ROS burden and, in turn, decreased the overexpressed p-AMPK. It is known that different pathways exist for AMPK activation, and the hypoxia-induced activation of AMPK is thought to be primarily driven by intracellular ROS rather than energy status [36]. The contrary effects of Rhodiola products for AMPK activation in different experimental conditions might be due to its adaptogen property, which normalizes body functions, allows the organism to adapt to environmental stresses, and prevents stressful insults from such factors [15, 37, 38]. Thus, Rhodiola species, which are phytoadaptogens, might act as bifunctional modulators for certain signal targets, such as AMPK, in response to different cellular conditions.

Although RCE and its bioactive components show excellent antioxidant potency, the expression level of the primary antioxidant enzymes, such as SOD2 and GPx2, was not changed in our experiment (Figure 3). These results are consistent with the previous study, which indicated that Rhodiola rosea can directly ameliorate the $\mathrm{H}_{2} \mathrm{O}_{2}$-induced oxidative stress but not through the activation of SOD and catalase [39]. Thus, our results imply that the antioxidant activity of Rhodiola species does not primarily result from the induction of antioxidant enzymes. These findings also agree with the great radical scavenging activity of RCE, salidroside, and tyrosol, as indicated by the elimination of the 2,2diphenyl-1-picrylhydrazyl (DPPH) radical, which is a stable radical that is widely used to test the efficacy of compounds as free-radical scavengers [19]. Thus, we suggest that RCE and its bioactive components attenuate the hypoxia-mediated oxidative stress via their function as ROS scavengers instead of through the induction of antioxidant enzymes. Combining the evidence from our cell and rodent models, we propose that the preventive effect of RCE, salidroside, and tyrosol for hypoxia-mediated Na,K-ATPase endocytosis is mediated through the alleviation of the generation of intracellular ROS, which in turn, decreases the p-AMPK level and PKC $\zeta$ translocation to the PM (Figure 6).

Alveolar hypoxia is not only a pathophysiological process in HAPE but also in other inflammatory-associated respiratory disorders, such as acute lung injury and acute respiratory distress syndrome, and the severity of these disorders is related to the dysfunction of Na,K-ATPase [9-11]. Similarly, hyperoxia-induced oxidative stress was also shown to inhibit Na,K-ATPase activity and AFC [7]. In the present study, Rhodiola products showed remarkable efficacy on $\alpha 1-\mathrm{Na}, \mathrm{K}-\mathrm{ATPa} e$ against hypoxic insults. Combined with anti-inflammatory activity [37], whether it has any beneficial effects on other hypoxia-associated 




FIGURE 6: The proposed mechanism of Rhodiola crenulata in attenuating the hypoxia-mediated Na,K-ATPase endocytosis. RCE and its bioactive components attenuate the hypoxia-induced $\alpha 1-\mathrm{Na}, \mathrm{K}$-ATPase endocytosis by inhibiting the ROS-AMPK-PKC $\zeta$ pathway.

respiratory disorders requires further elucidation, as there are many other ion channels that are known to be involved in transalveolar fluid transport, including $\mathrm{ENaC}[1,4]$. Thus, it would be interesting to investigate whether RCE is effective with other ion channels or against hyperoxia-impaired $\mathrm{Na}, \mathrm{K}$ ATPase function. It should be noted that the present study was carried out with some limitations, such as the fact that the measurement of ROS production as hypoxia-induced ROS production diminished after reoxygenation for 30 minutes [13]. Thus, the measurement needs to be processed as soon as possible in order to avoid disturbance due to reoxygenation. In addition, we showed that Rhodiola products and NAC almost completely alleviated the hypoxia-induced ROS production (Figures $2(\mathrm{a}), 2(\mathrm{~b})$, and 2(c)), but they restored only about $80-90 \%$ of $\mathrm{Na}, \mathrm{K}-\mathrm{ATP}$ ase expression (Figures $3(\mathrm{~b}$ ), $3(\mathrm{c})$, and $3(\mathrm{~d}))$. Combined with the results of compound C and GF10923X (Figure 4(d)), the findings indicated that blocking ROS-AMPK-PKC $\zeta$ pathway is not able to blunt $\mathrm{Na}, \mathrm{K}-\mathrm{ATPase}$ endocytosis completely. Our results suggest that ROS-AMPK-PKC $\zeta$ is the major, but not the only, signal pathway involved in the hypoxia-reduced $\mathrm{Na}, \mathrm{K}-\mathrm{ATPa} e$ endocytosis. However, it still needs to be further elucidated.

In conclusion, the present study provides significant evidence to support the ethnopharmacological role of Rhodiola crenulata as a popular folk medicine for high-altitude sickness in Tibet, and this effect is at least partly due to the ability to maintain pulmonary sodium transport via the preservation of $\mathrm{Na}$,K-ATPase.

\section{Conflict of Interests}

The authors declare that no competing financial interests exist.

\section{Acknowledgments}

This work was supported by Grants from the National Science Council (NSC 100-2320-B-016-007- and NSC 101-2320-B016-010-MY2 to Tsu-Chung Chang) and the Ministry of National Defense (DOD-101-14-5 to Tsu-Chung Chang and DOD-101-93 to Shih-Yu Lee), Taipei, Taiwan, ROC. The authors also thank Professor Daniel Steve Villarreal, Ph.D., Center for General Education, National Defense Medical Center, Taipei, Taiwan for the English editing.

\section{References}

[1] G. Zhou, L. A. Dada, and J. I. Sznajder, "Regulation of alveolar epithelial function by hypoxia," European Respiratory Journal, vol. 31, no. 5, pp. 1107-1113, 2008.

[2] H. Mortimer, S. Patel, and A. J. Peacock, "The genetic basis of high-altitude pulmonary oedema," Pharmacology and Therapeutics, vol. 101, no. 2, pp. 183-192, 2004.

[3] P. Hackett and D. Rennie, "High-altitude pulmonary edema," Journal of the American Medical Association, vol. 287, no. 17, pp. 2275-2278, 2002.

[4] H. Mairbäurl, "Role of alveolar epithelial sodium transport in high altitude pulmonary edema (HAPE)," Respiratory Physiology and Neurobiology, vol. 151, no. 2-3, pp. 178-191, 2006.

[5] P. Icard and G. Saumon, "Alveolar sodium and liquid transport in mice," American Journal of Physiology, vol. 277, no. 6, pp. L1232-L1238, 1999.

[6] M. R. Looney, C. Sartori, S. Chakraborty, P. F. James, J. B. Lingrel, and M. A. Matthay, "Decreased expression of both the $\alpha 1$ and $\alpha 2$-subunits of the Na-K-ATPase reduces maximal alveolar epithelial fluid clearance," American Journal of Physiology, vol. 289, no. 1, pp. L104-L110, 2005. 
[7] P. Factor, F. Saldias, K. Ridge et al., "Augmentation of lung liquid clearance via adenovirus-mediated transfer of a $\mathrm{Na}, \mathrm{K}$ ATPase $\beta 1$ subunit gene," Journal of Clinical Investigation, vol. 102, no. 7, pp. 1421-1430, 1998.

[8] J. Litvan, A. Briva, M. S. Wilson, G. R. S. Budinger, J. I. Sznajder, and K. M. Ridge, " $\beta$-Adrenergic receptor stimulation and adenoviral overexpression of superoxide dismutase prevent the hypoxia-mediated decrease in $\mathrm{Na}, \mathrm{K}$-ATPase and alveolar fluid reabsorption," Journal of Biological Chemistry, vol. 281, no. 29, pp. 19892-19898, 2006.

[9] I. Vadász, S. Raviv, and J. I. Sznajder, "Alveolar epithelium and $\mathrm{Na}, \mathrm{K}-\mathrm{ATP}$ ase in acute lung injury," Intensive Care Medicine, vol. 33, no. 7, pp. 1243-1251, 2007.

[10] I. T. Helenius, L. A. Dada, and J. I. Sznajder, "Role of ubiquitination in Na,K-ATPase regulation during lung injury," Proceedings of the American Thoracic Society, vol. 7, no. 1, pp. 65-70, 2010.

[11] M. A. Gropper and J. Wiener-Kronish, "The epithelium in acute lung injury/acute respiratory distress syndrome," Current Opinion in Critical Care, vol. 14, no. 1, pp. 11-15, 2008.

[12] C. Sartori, Y. Allemann, H. Duplain et al., "Salmeterol for the prevention of high-altitude pulmonary edema," New England Journal of Medicine, vol. 346, no. 21, pp. 1631-1636, 2002.

[13] L. A. Dada, N. S. Chandel, K. M. Ridge, C. Pedemonte, A. M. Bertorello, and J. I. Sznajder, "Hypoxia-induced endocytosis, of Na,K-ATPase in alveolar epithelial cells is mediated by mitochondrial reactive oxygen species and PKC- $\zeta$," Journal of Clinical Investigation, vol. 111, no. 7, pp. 1057-1064, 2003.

[14] G. A. Gusarova, L. A. Dada, A. M. Kelly et al., " $\alpha 1$-AMP-activated protein kinase regulates hypoxia-induced $\mathrm{Na}, \mathrm{K}$-ATPase endocytosis via direct phosphorylation of protein kinase C $\zeta$," Molecular and Cellular Biology, vol. 29, no. 13, pp. 3455-3464, 2009.

[15] A. Panossian, G. Wikman, and J. Sarris, "Rosenroot (Rhodiola rosea): traditional use, chemical composition, pharmacology and clinical efficacy," Phytomedicine, vol. 17, no. 7, pp. 481-493, 2010.

[16] B. C. Zhang, W. M. Li, R. Guo, and Y. W. Xu, "Salidroside decreases atherosclerotic plaque formation in low-density lipoprotein receptor-deficient mice," Evidence-Based Complementary and Alternative Medicine, vol. 2012, Article ID 607508, 5 pages, 2012.

[17] L. Sun, C. K. Isaak, and Y. Zhou, "Salidroside and Tyrosol from Rhodiola protect H9c2 cell from ischemia/reperfusion-induced apoptosis," Life Sciences, vol. 91, no. 5-6, pp. 151-158.

[18] S.-Y. Lee, M.-H. Li, L.-S. Shi et al., "Rhodiola crenulata extract alleviates hypoxic pulmonary edema in rats," Evidence-Based Complementary and Alternative Medicine, vol. 2013, Article ID 718739, 9 pages, 2013.

[19] D. Chen, J. Fan, P. Wang et al., "Isolation, identification and antioxidative capacity of water-soluble phenylpropanoid compounds from Rhodiola crenulata," Food Chemistry, vol. 134, no. 4, pp. 2126-2133, 2012.

[20] K. J. Nho, J. M. Chun, and H. K. Kim, "Ethanol extract of Dianthus chinensis L. induces apoptosis in human hepatocellular carcinoma HepG2 cells in vitro," Evidence-Based Complementary and Alternative Medicine, vol. 2012, Article ID 573527, 8 pages, 2012.

[21] F. N. Muanda, J. Bouayed, A. Djilani, C. Yao, R. Soulimani, and A. Dicko, "Chemical composition and, cellular evaluation of the antioxidant activity of Desmodium adscendens leaves," Evidence-Based Complementary and Alternative Medicine, vol. 2011, Article ID 620862, 9 pages, 2011.
[22] S. Nishiumi and H. Ashida, "Rapid preparation of a plasma membrane fraction from adipocytes and muscle cells: application to detection of translocated glucose transporter 4 on the plasma membrane," Bioscience, Biotechnology and Biochemistry, vol. 71, no. 9, pp. 2343-2346, 2007.

[23] J. Purushothaman, G. Suryakumar, D. Shukla et al., "Modulation of hypoxia-induced pulmonary vascular leakage in rats by seabuckthorn (Hippophae rhamnoides L.)," Evidence-Based Complementary and Alternative Medicine, vol. 2011, Article ID 574524, 13 pages, 2011.

[24] S. Cui, X. Hu, X. Chen, and Z. Hu, "Determination of ptyrosol and salidroside in three samples of Rhodiola crenulata and one of Rhodiola kirilowii by capillary zone electrophoresis," Analytical and Bioanalytical Chemistry, vol. 377, no. 2, pp. 370374, 2003.

[25] E. K. J. Pauwels, "The protective effect of the mediterranean diet: focus on cancer and cardiovascular risk," Medical Principles and Practice, vol. 20, no. 2, pp. 103-111, 2011.

[26] A. Tafesh, N. Najami, J. Jadoun, F. Halahlih, H. Riepl, and H. Azaizeh, "Synergistic antibacterial effects of polyphenolic compounds from olive mill wastewater," Evidence-based Complementary and Alternative Medicine, vol. 2011, Article ID 431021, 9 pages, 2011.

[27] S. A. Güney, A. Ott, S. Höschele et al., "Dexamethasone prevents transport inhibition by hypoxia in rat lung and alveolar epithelial cells by stimulating activity and expression of $\mathrm{Na}+$ $\mathrm{K}+$-ATPase and epithelial Na+ channels," American Journal of Physiology, vol. 293, no. 5, pp. L1332-L1338.

[28] R. Wodopia, H. S. Ko, J. Billian, R. Wiesner, P. Bärtsch, and H. Mairbäurl, "Hypoxia decreases proteins involved in epithelial electrolyte transport in A549 cells and rat lung," American Journal of Physiology, vol. 279, no. 6, pp. L1110-L1119, 2000.

[29] A. P. Comellas, L. A. Dada, E. Lecuona et al., "Hypoxiamediated degradation of $\mathrm{Na}, \mathrm{K}-\mathrm{ATP}$ ase via mitochondrial reactive oxygen species and the ubiquitin-conjugating system," Circulation Research, vol. 98, no. 10, pp. 1314-1322, 2006.

[30] I. Vadász, L. A. Dada, A. Briva et al., "AMP-activated protein kinase regulates $\mathrm{CO}_{2}$-induced alveolar epithelial dysfunction in rats and human cells by promoting $\mathrm{Na}, \mathrm{K}-\mathrm{ATPase}$ endocytosis," Journal of Clinical Investigation, vol. 118, no. 2, pp. 752-762, 2008.

[31] X. Chen, Q. Zhang, Q. Cheng, and F. Ding, "Protective effect of salidroside against $\mathrm{H}_{2} \mathrm{O}_{2}$-induced cell apoptosis in primary culture of rat hippocampal neurons," Molecular and Cellular Biochemistry, vol. 332, no. 1-2, pp. 85-93, 2009.

[32] C.-B. Tan, M. Gao, W.-R. Xu, X.-Y. Yang, X.-M. Zhu, and G.-H. $\mathrm{Du}$, "Protective effects of salidroside on endothelial cell apoptosis induced by cobalt chloride," Biological and Pharmaceutical Bulletin, vol. 32, no. 8, pp. 1359-1363, 2009.

[33] O. Koksel, A. Ozdulger, M. Ercil et al., "Effects of N-acetylcysteine on oxidant-antioxidant balance in oleic acid-induced lung injury," Experimental Lung Research, vol. 30, no. 6, pp. 431446, 2004.

[34] Z. Liu, X. Li, A. R. Simoneau, M. Jafari, and X. Zi, "Rhodiola rosea extracts and salidroside decrease the growth of bladder cancer cell lines via inhibition of the mTOR pathway and induction of autophagy," Molecular Carcinogenesis, vol. 51, no. 3, pp. 257-267, 2012.

[35] H.-B. Li, Y.-K. Ge, X.-X. Zheng, and L. Zhang, "Salidroside stimulated glucose uptake in skeletal muscle cells by activating AMP-activated protein kinase," European Journal of Pharmacology, vol. 588, no. 2-3, pp. 165-169, 2008. 
[36] B. M. Emerling, F. Weinberg, C. Snyder et al., "Hypoxic activation of AMPK is dependent on mitochondrial ROS but independent of an increase in AMP/ATP ratio," Free Radical Biology and Medicine, vol. 46, no. 10, pp. 1386-1391, 2009.

[37] P. A. S. Bawa and F. Khanum, "Anti-inflammatory activity of Rhodiola rosea - "A second-generation adaptogen"', Phytotherapy Research, vol. 23, no. 8, pp. 1099-1102, 2009.

[38] D. Seely and R. Singh, "Adaptogenic potential of a polyherbal natural health product: report on a longitudinal clinical trial," Evidence-Based Complementary and Alternative Medicine, vol. 4, no. 3, pp. 375-380, 2007.

[39] M. M. Bayliak and V. I. Lushchak, "The golden root, Rhodiola rosea, prolongs lifespan but decreases oxidative stress resistance in yeast Saccharomyces cerevisiae," Phytomedicine, vol. 18, no. 14, pp. 1262-1268, 2011. 


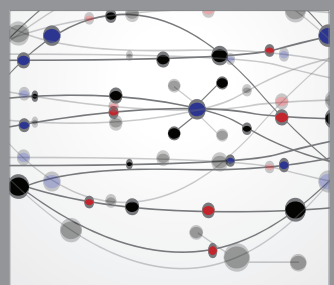

The Scientific World Journal
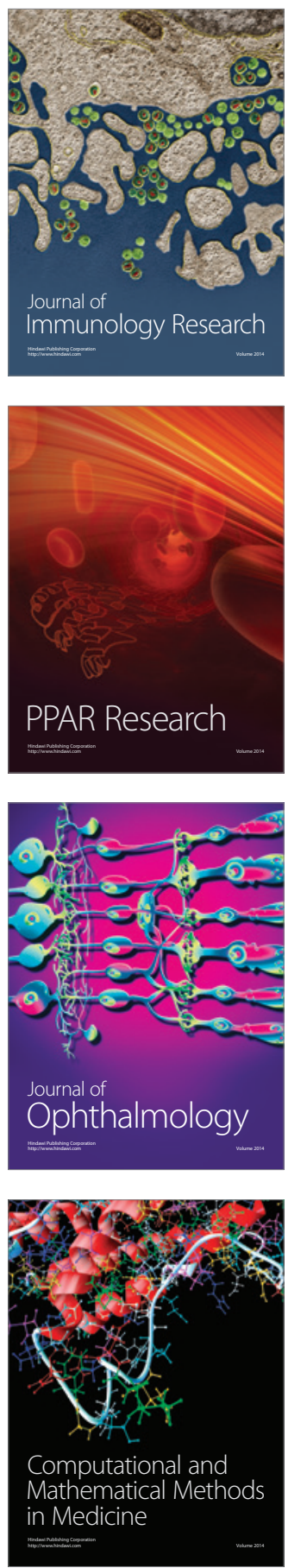

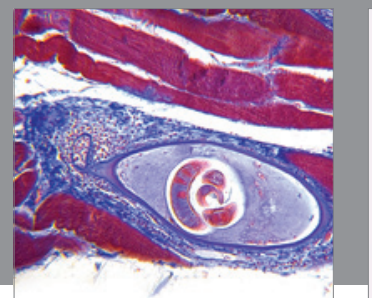

Gastroenterology

Research and Practice
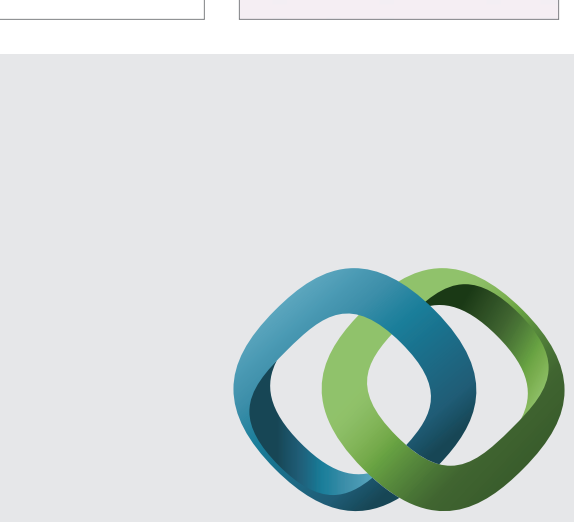

\section{Hindawi}

Submit your manuscripts at

http://www.hindawi.com
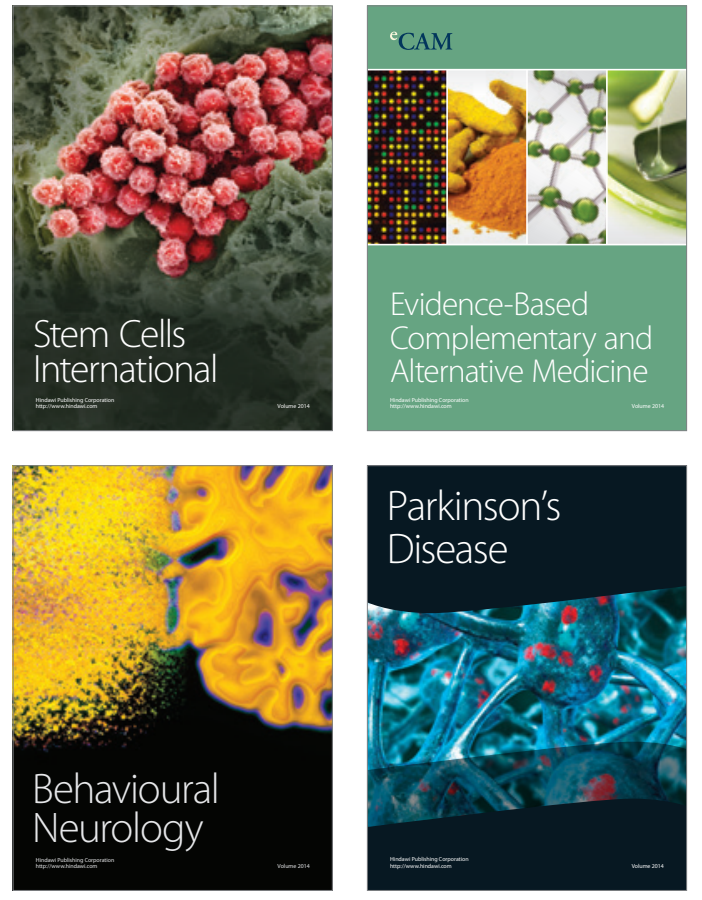
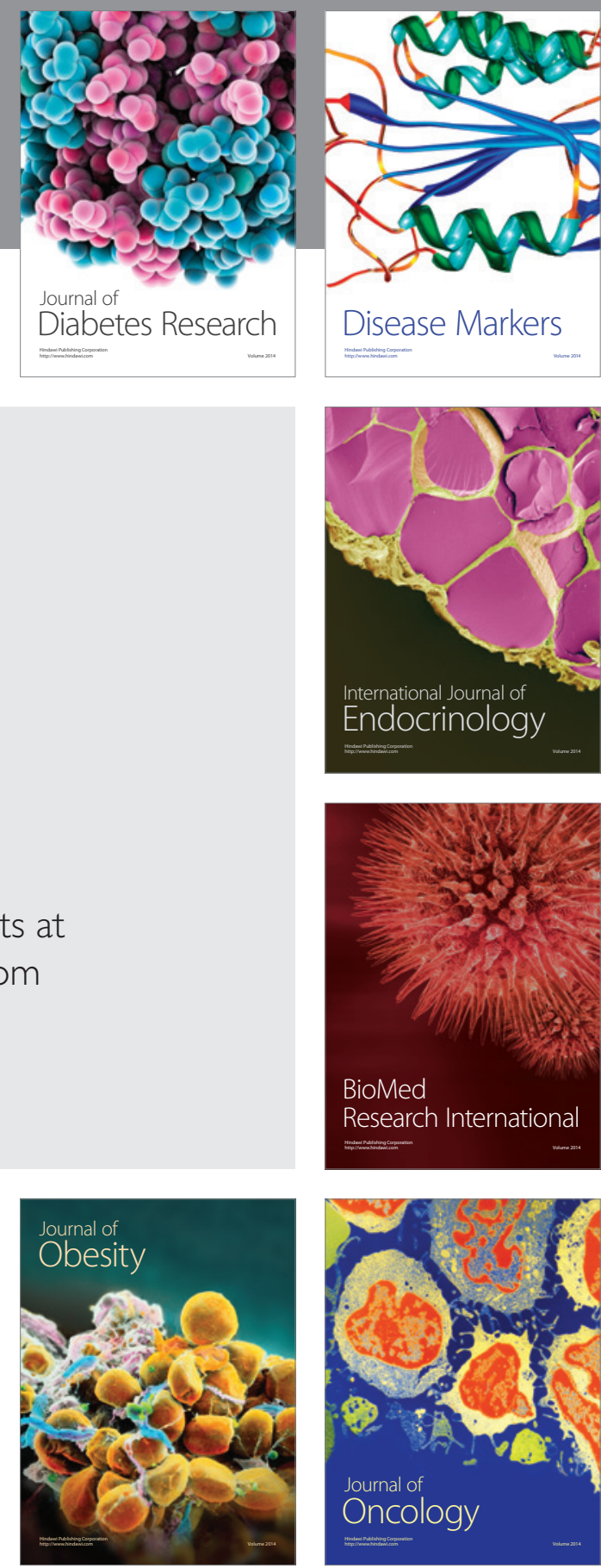

Disease Markers
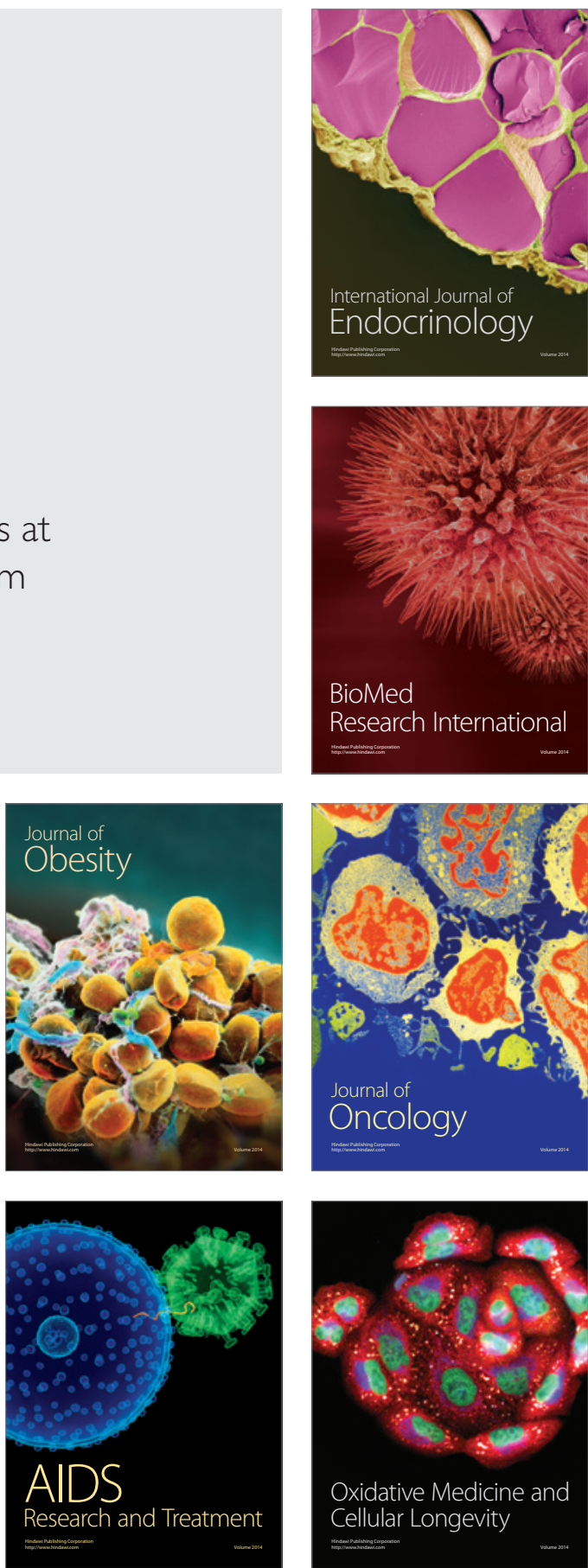